FisioterBras 2022;23(1):18-36

doi: $\underline{10.33233 / \mathrm{fb} . v 23 i 1.4921}$

\title{
ARTIGO ORIGINAL \\ Percepção dos discentes de fisioterapia acerca de doenças infectocontagiosas: uma pesquisa de campo
}

Perception of physical therapy students about infectious and contagious diseases: a field research

Joice Sousa Santos ${ }^{*}$, Ohanna Cruz Pereira*, Mariana Machado dos Santos*, Leila Brito de Queiroz Ribeiro, D.Sc. ${ }^{* *}$, Alena Ribeiro Alves Peixoto Medrado D.Sc. ${ }^{* * *}$

*Graduandas em Fisioterapia pela Universidade Federal da Bahia, **Professora Adjunta do Curso de Odontologia da Escola Bahiana de Medicina e Saúde Pública, ${ }^{* * *}$ Professora Adjunta da Universidade Federal da Bahia

Recebido em 13 de setembro de 2021; aceito em 18 de janeiro de 2022.

Correspondência: Alena Ribeiro Alves Peixoto Medrado, Avenida Reitor Miguel Calmon s/no., Vale do Canela, 40000-000 Salvador BA

Joice Sousa Santos: joice.sousa@ufba.br, Ohanna Cruz Pereira: ohanna.cruz@ufba.br Mariana Machado dos Santos: marianamachado1305@gmail.com Leila Brito de Queiroz Ribeiro: leilabqueiroz@yahoo.com.br Alena Ribeiro Alves Peixoto Medrado: armedrado@ufba.br

\section{Resumo}

Introdução: As atividades desenvolvidas nos estabelecimentos de saúde muitas vezes constituem um risco à saúde dos profissionais e pacientes, principalmente em relação às doenças infectocontagiosas. Nesse contexto, sabe-se que os pacientes atendidos pelo profissional fisioterapeuta variam desde atletas a indivíduos imunossuprimidos, o que torna imprescindível a inserção desta temática no currículo dos estudantes. Objetivo: Investigar a percepção dos estudantes de Fisioterapia de uma universidade pública acerca de conceitos de biossegurança e algumas doenças infectocontagiosas. Métodos: Tratou-se de um estudo quali-quantitativo do tipo descritivo exploratório de caráter transversal, no qual foram aplicados questionários sobre a conduta do fisioterapeuta frente às doenças infectocontagiosas. A amostra, definida por conveniência, foi composta por 105 estudantes. Resultados: Cerca de 67,3\% dos estudantes 
reconheceram o conceito de biossegurança. Em relação às precauções de contato, 59\% dos discentes afirmaram serem necessárias em casos de escabiose, 46,7\% na furunculose e 34,3\% no impetigo. Conclusão: A partir do presente estudo, foi possível concluir que apesar do elevado percentual de respostas assertivas, o aprendizado adquirido durante a formação acadêmica pode ser perdido no decorrer das práticas ocupacionais, o que demonstra a importância da educação continuada na prática clínica do profissional fisioterapeuta.

Palavras-chave: Fisioterapia; contenção de riscos biológicos; doenças transmissíveis.

\begin{abstract}
Introduction: The activities carried out in health establishments often pose a risk to the health of professionals and patients, especially in relation to infectious and contagious diseases. In this context, it is known that patients cared for by professional physical therapists vary from athletes to immunosuppressed individuals, which makes it essential to include this theme in the students' curriculum. Objective: To investigate the perception of physical therapy students at a public University about concepts of biosafety and some infectious diseases. Methods: This was a qualitative-quantitative, descriptive, exploratory, cross-sectional study, in which questionnaires were applied on the physical therapist's conduct in relation to infectious and contagious diseases. The sample, defined by convenience, consisted of 105 students. Results: About $67.3 \%$ of students recognized the concept of biosafety. Regarding contact precautions, $59 \%$ of students said they were necessary in cases of scabies, $46.7 \%$ in furunculosis and $34.3 \%$ in impetigo. Conclusion: From the present study, it was possible to conclude that despite the high percentage of assertive answers, the learning acquired during academic training may be lost during occupational practices, which demonstrates the importance of continuing education in the clinical practice of physical therapists.
\end{abstract}

Keywords: Physical Therapy Specialty, containment of biohazards; communicable diseases.

\title{
Introdução
}

Os estabelecimentos de saúde como unidades básicas e hospitais, assim como as atividades desempenhadas nesses ambientes, muitas vezes constituem um risco à saúde dos profissionais e pacientes inseridos nos locais de assistência [1]. Dentre esses riscos, os de natureza biológica ganham destaque, pois caracterizam-se como a principal forma de exposição do profissional de saúde no cuidado ao paciente [2]. Entre as medidas voltadas para diminuir essas ameaças, pode-se citar o conhecimento acerca 
de procedimentos e normas de biossegurança, as quais preconizam a informação em relação aos riscos aos quais o profissional está exposto, principalmente a forma como a contaminação pode ocorrer [3].

De acordo com o Ministério da Saúde, as doenças infectocontagiosas consistem em patologias nas quais ocorre a infecção de um organismo hospedeiro por um determinado patógeno que se multiplica a partir da utilização de recursos desse hospedeiro. Ademais, é capaz de modificar a fisiologia normal do organismo e, consequentemente, acarretar prejuízos à saúde do indivíduo [4].

Os hospitais são espaços onde há número considerável de pacientes portadores dessas doenças, como meningite, tuberculose, Aids, hanseníase, hepatite, sarampo e mais recentemente, a COVID-19, o que faz desses locais espaços onde ocorre uma concentração significativa de microrganismos patogênicos $[5,6]$. Isso pode levar ao aumento substancial de infecções hospitalares, as quais são um dos fatores determinantes de morbimortalidade entre pacientes hospitalizados [7]. Por conta do número elevado de infecções no ambiente hospitalar por microrganismos que desenvolveram resistência a agentes antimicrobianos, surge a necessidade crescente da adoção de medidas preventivas. Por essa razão, a Comissão de Controle de Infecções Hospitalares ( $\mathrm{CCIH}$ ) é exigida nos hospitais; ela também é responsável pela realização de políticas de prevenção e controle de transmissão de agentes infecciosos a pacientes e trabalhadores no ambiente hospitalar [8,9].

Em relação à Fisioterapia, essa caracteriza-se como uma profissão que vem crescendo no Brasil. A grade curricular ofertada por esse curso é extensa e apresenta um leque diversificado de disciplinas, inclusive aquelas que contemplam a área de conhecimento da Microbiologia e Imunologia. Vale ressaltar que alguns profissionais fisioterapeutas estarão inseridos em hospitais e, consequentemente, precisarão do conhecimento sobre as infecções que comumente acontecem nesses ambientes, com o objetivo de mitigar ou prevenir os riscos durante o exercício de sua profissão.

Sabe-se também que a condição de saúde dos pacientes atendidos pelos fisioterapeutas varia bastante, incluindo indivíduos imunossuprimidos [10]. Assim, os planos para adoção de procedimentos de controle de infecção devem abarcar essa variedade de pacientes e a possibilidade de disseminar doenças transmissíveis para essas populações mais vulneráveis [11]. Entretanto, apesar de a abordagem desse conteúdo no currículo dos cursos de Fisioterapia ser imprescindível, percebe-se que a maior atenção dos discentes está voltada para as disciplinas profissionalizantes em detrimento das áreas de concentração mais abrangentes, a exemplo das disciplinas básicas que são compartilhadas por diferentes matrizes curriculares de cursos da saúde. 
Diante do que foi apresentado, o objetivo do presente estudo foi investigar a percepção dos estudantes de Fisioterapia de uma universidade pública acerca de conceitos de biossegurança e algumas doenças infectocontagiosas.

\section{Métodos}

Estratégia de coleta de dados

Foi realizado um estudo quali-quantitativo do tipo descritivo exploratório de caráter transversal, a partir da coleta de dados através de questionário físico e online acerca da conduta do fisioterapeuta frente às doenças infectocontagiosas, no período de junho de 2019 a maio de 2020, na população de estudantes do curso de Fisioterapia da Universidade Federal da Bahia (UFBA) no Instituto de Ciências da Saúde, Salvador/BA.

A aprovação do Comitê de Ética em Pesquisa do Instituto de Ciências da Saúde da Universidade Federal da Bahia foi concedida para a realização do presente estudo, assim como a aprovação em conformidade com a Resolução № 466/2012, do Conselho Nacional de Saúde/MS, a qual dispõe sobre a pesquisa e os testes envolvendo seres humanos, através do número de protocolo 3.307.477.

O desenvolvimento do estudo ocorreu a partir da elaboração de um questionário adaptado com perguntas objetivas, o qual foi aplicado por um entrevistador previamente calibrado. Ademais, houve também a aplicação do questionário através de plataforma digital (Google Forms).

\section{População e amostra}

A população utilizada no estudo foi formada por uma amostra de conveniência que abrangeu 105 graduandos de diferentes semestres do curso de Fisioterapia da UFBA que aceitaram participar do estudo e informaram este consentimento através de assinatura no Termo de Consentimento Livre e Esclarecido. Estes preencheram o instrumento de pesquisa sem interferências externas e em horário disponível para cada participante. Não houve desistências após a autorização da participação.

Os critérios de inclusão aplicam-se a estudantes do curso de Fisioterapia da UFBA, regularmente matriculados no curso, maiores de 18 anos que estivessem cursando o primeiro, sexto ou décimo semestres, por serem períodos representativos do início, meio e fim do curso, respectivamente. Discentes que haviam efetuado o 
trancamento da matrícula ou aqueles transferidos de outras universidades não foram incluídos no estudo.

\section{Protocolo do estudo}

Os dados foram coletados através de questionários presenciais e do envio de formulários online através da plataforma Google Forms, sendo esses roteiros semiestruturados e adaptados com perguntas de caráter objetivo e subjetivo, as quais foram validadas anteriormente por Moreira [3]. Esse questionário foi baseado também no que foi aplicado por Campos [12], e no Manual de Legislação de Segurança e Medicina no Trabalho [13].

\section{Análise dos resultados e estatística}

Foram utilizados gráficos e tabelas para organizar os dados obtidos, apresentando frequência absoluta e relativa em planilhas no Word/ Excel 2010. Foi utilizado o software R (versão 4.0.3), no qual foi feita uma análise descritiva (frequência absoluta/relativa, média e desvio padrão) a fim de identificar tanto as características gerais quanto as específicas da amostra estudada.

\section{Resultados}

A amostra foi composta por 105 graduandos do curso de Fisioterapia, dos quais $75,2 \%$ ( $n=79)$ eram do sexo feminino e $24,8 \%(n=26)$, do sexo masculino.

A tabela I ilustra como a biossegurança foi definida pelos participantes. Observou-se que $67,3 \%(n=70)$ dos estudantes reconheceram que a biossegurança representa um conjunto de ações voltadas para a prevenção, minimização ou eliminação dos riscos que podem comprometer a saúde do homem e animais, o meio ambiente ou a qualidade dos trabalhos desenvolvidos, durante as atividades de pesquisa, produção, ensino, desenvolvimento tecnológico e prestação de serviços. Dos discentes entrevistados, $19,2 \%(n=20)$ afirmaram que a biossegurança é um conjunto de normas para a prevenção, minimização ou eliminação dos riscos que podem comprometer a saúde do trabalhador durante as atividades de serviço em ambiente hospitalar, ao passo que $13,5 \%(n=14)$ se referiram à biossegurança como sendo apenas a utilização de equipamentos de proteção individual e coletivo, visando a proteção e qualidade de vida dos trabalhadores de saúde durante sua atividade profissional. 
Tabela I - Frequência absoluta e relativa de discentes do curso de Fisioterapia em relação ao conceito de Biossegurança, Instituto de Ciências da Saúde, Universidade Federal da Bahia, Salvador/BA, 2020

\begin{tabular}{llc}
\hline Conceito Biossegu rança & Frequência absoluta & $\begin{array}{l}\text { F requência } \\
\text { relativa }\end{array}$ \\
\hline $\begin{array}{l}\text { Conjunto de ações voltadas para a prevenção, } \\
\text { minimização ou eliminação dos riscos que }\end{array}$ & N & $\%$ \\
podem comprometer a saúde do homem e \\
animais, o meio ambiente ou a qualidade dos \\
trabalhos desenvolvidos, durante as atividades \\
de pesquisa, produção, ensino, \\
$\begin{array}{l}\text { deservolvimento tecnológico e prestação de } \\
\text { serviços }\end{array}$ \\
$\begin{array}{l}\text { Conjunto de normas para a prevenção, } \\
\text { minimização ou eliminação dos riscos que } \\
\text { podem comprometer a saúde do trabalhador } \\
\text { durante as atividades de serviço em ambiente } \\
\text { hospitalar }\end{array}$ \\
$\begin{array}{l}\text { Trata-se apenas da utilização de equipamentos } \\
\text { de proteção individual e coletivo, visando à } \\
\text { proteção e qualidade de vida dos } \\
\text { trabalhadores de saúde durante sua atividade } \\
\text { profissional }\end{array}$ \\
\hline Total
\end{tabular}

*1 dado perdido. Fonte: Autoria própria

O gráfico I ilustra o percentual de alunos que afirmaram conhecer a Norma Reguladora (NR) 32. Foi constatado que 87,6\% $(n=92)$ dos entrevistados descreveram a NR 32 como uma norma regulamentadora que estabelece as diretrizes básicas para a implementação de medidas de proteção à segurança e à saúde dos trabalhadores em serviços de saúde. Cerca de 7,6\% $(n=8)$ dos estudantes responderam que a NR 32 se tratava de um órgão regulamentador que tem como propósito garantir que os trabalhadores de saúde apresentem cuidados quanto aos procedimentos de atendimento, com qualidade e segurança no trabalho. Adicionalmente, $4,8 \%$ dos discentes identificaram a NR 32 como um instrumento do qual os trabalhadores dispõem para tratar da prevenção de acidentes de trabalho, das condições do ambiente de trabalho e de todos os aspectos que afetam sua saúde e segurança.

O gráfico II ilustra que $96,2 \%(n=101)$ dos participantes entrevistados concordaram com a aplicação de procedimentos de precaução padrão para todos os pacientes independente do diagnóstico, enquanto que $3,8 \%(n=4)$ dos estudantes responderam que tais medidas protetivas não são necessárias. 




Fonte: Autoria própria

Gráfico I - Percentual de discentes do curso de Fisioterapia em relação à percepção sobre o conceito da NR 32, Instituto de Ciências da Saúde, Universidade Federal da Bahia, Salvador/BA, 2020

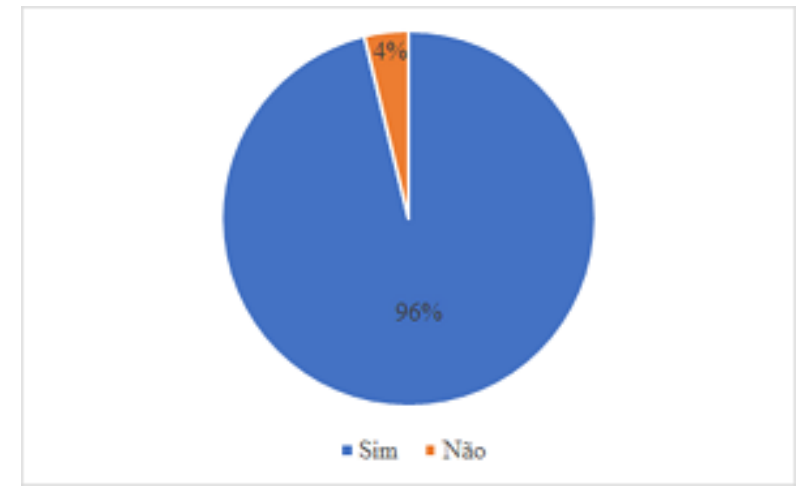

Fonte: Autoria própria

Gráfico II - Frequência relativa de discentes do curso de Fisioterapia em relação à utilização de precaução padrão em quaisquer situações, independente do diagnóstico, Instituto de Ciências da Saúde, Universidade Federal da Bahia, Salvador/BA, 2020

A tabela Il descreve a identificação das doenças infecciosas pelos discentes para as quais são necessárias medidas de precaução de contato. Na amostra avaliada, 59\% ( $n=62)$ dos estudantes reconheceram a escabiose como uma doença que os fisioterapeutas devem tomar cuidado e adotar tais medidas, seguida pela furunculose (47\%; $n=49)$, HIV (38\%, $n=40)$ e tuberculose $(37 \%, n=39)$. A dengue foi a condição menos indicada pelos participantes para a adoção de medidas de precaução de contato. (8\%, $n=8)$. 
Tabela II - Frequência absoluta e relativa de discentes do curso de Fisioterapia em relação à identificação das patologias com as quais devem ser utilizadas precaução de contato, Instituto de Ciências da Saúde, Universidade Federal da Bahia, Salvador/BA, 2020

\begin{tabular}{lcc}
\hline Patologia & Frequência absoluta & Frequência relativa \\
\hline Escabiose & $\mathbf{N}$ & $\%$ \\
Furunculose & 62 & 59 \\
HIV & 49 & 46,7 \\
Tuberculose & 40 & 38,1 \\
Impetigo & 39 & 37,1 \\
Hepatite & 36 & 34,3 \\
Meningite & 30 & 28,6 \\
Dengue & 27 & 25,7 \\
Total & 8 & 7,6 \\
\hline
\end{tabular}

Fonte: Autoria própria

A tabela III ilustra a frequência absoluta e relativa de discentes que identificaram os Equipamentos de Proteção Individual (EPIs). As luvas foram apontadas pela maioria dos estudantes como um EPI (99\%, $n=104)$, assim como os óculos $(94,3 \%, n=99)$ e o jaleco $(93,3 \%, n=98)$. Já a máscara $(78,1 \%, n=82)$ e a touca $(73,3 \%, n=77)$ foram identificados com uma frequência menor. Os propés foram os EPIs menos destacados pelos estudantes $(58,1 \%, \mathrm{n}=61)$.

Tabela III - Frequência absoluta e relativa de discentes do curso de Fisioterapia em relação à identificação dos Equipamentos de Proteção Individual, Instituto de Ciências da Saúde, Universidade Federal da Bahia, Salvador/BA, 2020

\begin{tabular}{llc}
\hline EPI & F requência absoluta & Frequência relativa \\
\hline Luva & $\mathbf{N}$ & $\%$ \\
Óculos & 104 & 99 \\
Jaleco & 99 & 94,3 \\
Máscara & 98 & 93,3 \\
Touca & 82 & 781 \\
Propés & 77 & 73,3 \\
\hline
\end{tabular}

Fonte: Autoria própria

\section{Discussão}

As práticas de biossegurança ganharam destaque nos últimos meses como nunca tinha ocorrido antes no cenário mundial [14]. Os profissionais de saúde, entre os quais se incluem os fisioterapeutas, devem estar atentos aos conceitos básicos de biossegurança, em especial no ambiente laboral. De acordo com a Agência Nacional de Vigilância Sanitária, a biossegurança consiste no conjunto de ações voltadas a diminuir ou eliminar os riscos relacionados às atividades que possam prejudicar a qualidade de vida e a saúde humana, além do meio ambiente [4]. No presente estudo, observou-se que um percentual expressivo dos discentes entrevistados $(67,3 \%)$ compreendeu 0 
conceito apresentado. O estudo de Schroeder et al. [15] realizou análise similar; contudo, esse se concentrou na visão dos acadêmicos de Odontologia. Observou-se que 75,35\% dos estudantes afirmaram que conheciam as normas de biossegurança, além de De Melo et al. [16] relatarem, em seu trabalho, que $82 \%$ dos estudantes do mesmo curso se autoavaliaram bons conhecedores desta temática. Outros autores também relataram resultados satisfatórios em outros cursos de graduação na área de saúde $[17,18]$. Percebeu-se uma escassez de estudos com análise voltada para alunos de graduação em Fisioterapia.

Segundo Gasperini [19], a biossegurança faz parte do dia a dia dos profissionais da Fisioterapia, assim como da prática e dos cuidados a nível hospitalar, os quais podem ocorrer na enfermaria, no ambulatório ou nas unidades de terapia intensiva. Assim, a implementação de práticas de biossegurança nos serviços de saúde ainda é um desafio, tornando-se necessário e imprescindível o apoio da gestão em fornecer capacitação contínua, a fim de minimizar os riscos ocupacionais e garantir tanto a segurança dos profissionais quanto a dos pacientes.

Já a Norma Regulamentadora de Segurança e Saúde no Trabalho em Serviços de Saúde (NR-32), publicada pelo Ministério do Trabalho e Emprego em 2005, considera as particularidades para controle e prevenção dos riscos inerentes aos ambientes onde são prestados os atendimentos de saúde [4]. Dentre os aspectos contemplados na NR-32, pode-se citar a necessidade de capacitação continuada, a importância das imunizações, os riscos biológicos, além da adoção de outras medidas voltadas para identificar e prevenir os riscos, com objetivo de garantir a saúde dos profissionais.

Em nossa pesquisa, 87,6\% dos discentes do curso de Fisioterapia declararam ter conhecimento da Norma Regulamentadora $n^{\circ} 32$ (NR-32). A importância do reconhecimento da NR-32 reside no fato de que a adoção das suas orientações previne patologias evitáveis [20]. Estudos apontam que muitos serviços de saúde não se adequaram devidamente às normas, pois há evidências do despreparo profissional e acidentes de trabalho. Nobre descreve os principais fatores para os profissionais não utilizarem a NR-32, os quais envolvem a falta de conhecimento da NR, falta de tempo, falta de EPIs e EPCs e falta de um programa para saúde do trabalhador [21]. Vale destacar que o nível de conhecimento depende do nível de instrução, do interesse pessoal e do tempo de estudo.

A Precaução Padrão (PP), por sua vez, é definida como o conjunto de estratégias adotadas para prevenir a exposição a materiais biológicos no ambiente de trabalho $[22,23]$. Essas estratégias são universais, ou seja, são aplicáveis a todos os profissionais de saúde em contato direto com pacientes, independente da suspeita ou 
diagnóstico prévio de doença infecciosa [24]. São necessárias especialmente quando há risco de contato com sangue e outros fluidos corpóreos [25]. Entre as principais medidas de precaução padrão, pode-se citar o uso correto de equipamentos de proteção individual (EPI), a higienização das mãos, o manuseio e o descarte adequado de instrumentos perfurocortantes, a descontaminação do local e equipamentos e o gerenciamento seguro de roupas e resíduos e imunização [22,24].

Hessels [24] descreveu em sua revisão que a maioria dos estudos determina a PP como uma construção global, apesar de possuir facilitadores e barreiras para maior adesão. No presente estudo, $96 \%$ dos discentes afirmaram que a aplicação das PPs é indispensável independente do diagnóstico, o que se assemelha ao resultado de Moreira, no qual foi unânime a resposta afirmativa para essa questão. Destaca-se a percepção da importância das PPs para proteção de contaminações a patógenos transmissíveis; por essa razão, existe a necessidade de que todos os profissionais possuam o conhecimento das formas de aplicação dessas medidas, o que irá auxiliar na redução de contaminações cruzadas, acidentes de trabalho e prejuízos institucionais [3]. Segundo o estudo de Oliveira [26], que verificou o conhecimento de profissionais de saúde da Unidade de Terapia Intensiva, constatou-se que apenas 36,3\% dos participantes apresentaram conhecimento apropriado em relação às normas de precaução padrão. Ainda nesse estudo, verificou-se que os fisioterapeutas obtiveram o menor percentual de conhecimento.

Existem também outras práticas mais específicas que devem ser implantadas quando as medidas de precaução padrão não são suficientes para a interrupção total da transmissão do agente infeccioso. Essas precauções estão relacionadas com a forma de transmissão, as quais variam entre precauções de contato, precaução para gotículas e precaução para aerossóis [27]. No que tange às precauções de contato, essas consistem em medidas que devem ser adotadas pelos profissionais durante 0 atendimento aos pacientes portadores de doenças infectocontagiosas, a fim de prevenir riscos à saúde, através do controle da disseminação de patógenos [28,29]. Essas medidas incluem a higienização das mãos, o uso do avental e de luvas, além do quarto privativo [29]. Tais medidas previnem tanto a contaminação direta, a qual ocorre através do contato físico entre o portador da infecção e o hospedeiro suscetível, quanto a contaminação indireta, caracterizada pelo contato de um possível hospedeiro com instrumentos e equipamentos contaminados [30].

A higienização das mãos é uma das práticas mais importantes no cuidado ao paciente, visto que a maior parte das transmissões de infecções ocorre através da mão dos profissionais de saúde [31,32]. Ademais, é uma medida fundamental para os profissionais fisioterapeutas, pois estes utilizam-nas constantemente nos seus 
atendimentos. É importante que a lavagem seja realizada de forma correta para impedir a transmissão de microrganismos no contato com qualquer paciente [3].

Já o avental caracteriza-se como um EPI, o qual protege as roupas e as superfícies corporais quando há risco de contaminação; ele deve ser utilizado em situações de contato direto com pacientes portadores de doenças transmissíveis através de contato, como a furunculose, o impetigo, a escabiose, entre outras. Esse EPI deve ser longo e possuir mangas compridas; deve ser descartável e estéril e sua colocação e retirada devem ser feitas na antessala ou no quarto, com posterior descarte em local adequado, seguido da lavagem das mãos [4].

As luvas, por sua vez, caracterizam-se por ser o EPI mais utilizado pelos profissionais, sendo imprescindíveis no contato com um paciente portador de doença infectocontagiosa [33]. Por fim, o quarto privativo é importante pois mantém o paciente isolado, o que dificulta a infecção entre outras pessoas; na ausência do quarto privativo, deve-se alocar os pacientes em enfermarias de coorte, nas quais são atendidos os casos em que houve infecção pelo mesmo agente patogênico [34]. Sugere-se também que os materiais utilizados, tais como estetoscópio, termômetro, esfigmomanômetro, entre outros, sejam de uso exclusivo do paciente, a fim de diminuir a contaminação indireta [29].

No presente estudo, 59\% dos discentes afirmaram ser necessária a adoção dessas práticas em pacientes portadores de escabiose. Em outro estudo, o qual verificou a adesão dos profissionais de saúde às precauções de contato em unidade de terapia intensiva, verificou-se que $41,9 \%$ dos participantes afirmaram tal necessidade em relação às dermatites e outras doenças de pele e mucosas, as quais incluem a escabiose [35]. Esses resultados diferem do que foi encontrado por Moreira [3], no qual apenas $23 \%$ dos discentes afirmaram serem necessárias tais medidas, e por Campos [36], que obteve 100\% de afirmações para essa infecção. Embora não seja considerada pelo Ministério da Saúde como doença ocupacional com consequências graves para profissionais de saúde, a escabiose é uma patologia que exige as precauções de contato no cuidado ao paciente, a fim de evitar a sua disseminação [37].

Em relação à furunculose, 46,7\% dos estudantes afirmaram a necessidade da adoção das precauções de contato. Esse resultado foi semelhante ao estudo de Campos, o qual apresentou índice de $42,9 \%$ para essa doença [36]. O desconhecimento associado a essa patologia foi visto também no estudo de Moreira [3], no qual apenas $16 \%$ dos discentes reconhecem a importância das práticas no cuidado aos pacientes infectados com a patologia. Isso pode se refletir em uma baixa adesão dessas medidas por parte dos futuros profissionais, o que pode resultar no aumento da transmissão entre pacientes e trabalhadores de saúde. 
O impetigo, por sua vez, foi apontado por apenas 34,3\% dos estudantes; no estudo de Moreira [3], o índice de respostas foi ainda menor, pois apenas $8 \%$ dos discentes considerou o impetigo como uma patologia que exige precaução de contato. Já Campos [36] encontrou um resultado próximo ao do presente artigo, com 42,9\% dos profissionais afirmando a necessidade desse tipo de precaução.

Quando perguntados sobre a importância das precauções de contato no cuidado aos pacientes portadores de HIV, 38,1\% dos discentes afirmaram que tais práticas são necessárias. Esse resultado apresentou índices maiores quando comparado a outros estudos. Na pesquisa realizada por Moreira [3], 16\% dos discentes afirmaram que há essa necessidade. O estudo de Garcia [35] apresentou resultados semelhantes, com 16,1\% dos estudantes apontando essa importância.

Apesar de pertencer à classe 3 e ser um agente que representa risco grave para o profissional exposto e moderado para a comunidade, com sinais clínicos e lesões graves, o HIV é um patógeno que não exige dos profissionais as precauções de contato. Além do HIV, o vírus da hepatite $\mathrm{B}$ e a tuberculose também se caracterizam como riscos ocupacionais que resultam em maiores consequências para o profissional [37]. De acordo com Carmagnani [38], os profissionais de saúde preocupam-se mais com a sua segurança pessoal quando o paciente é portador dessas patologias em detrimento de outras que também necessitam de cuidados. Isso contribui para a adoção de práticas equivocadas e, consequentemente, para o aumento da transmissão e dos índices de infecção hospitalar.

Quanto ao alto risco de contaminação pelo HIV, outras pesquisas apontaram um baixo nível de conhecimento dos profissionais de saúde. Conforme Duarte [39], o qual analisou os fatores de riscos ocupacionais do trabalho de enfermagem sob a ótica dos enfermeiros, cerca de $75,7 \%$ dos profissionais não sabe qual conduta realizar em casos de exposição a sangue e/ou líquidos corpóreos, o que pode contribuir para o aumento dos níveis de transmissão desse vírus. Entretanto, esse risco pode ser nulo ao se utilizar luvas de forma adequada durante o cuidado ao paciente, o que ainda não é observado na prática por muitos profissionais de saúde [39,40].

Em relação à tuberculose, $37,1 \%$ dos participantes apontaram a necessidade das precauções de contato no cuidado com esses pacientes. Esse resultado foi mais próximo ao encontrado por Moreira [3], no qual 16\% dos estudantes fizeram tal afirmação. Outras pesquisas apresentaram índices maiores de respostas para esse tópico, sendo 54,8\% no artigo de Garcia [35] e 78,6\% no de Campos [36]. Essas diferenças refletem a falta de conhecimento acerca das medidas adequadas, pois, de acordo com esses estudos, as práticas voltadas para pacientes com tuberculose relacionam-se às precauções padrão e por aerossóis. Entretanto, vale destacar a 
importância do quarto privativo com ventilação especial através da filtração do ar com filtro de alta eficiência (HEPA - High Efficiency Particulate Air) e da aplicação da pressão negativa, com o objetivo de evitar a contaminação por essa patologia [37].

No que diz respeito à hepatite, $28,6 \%$ declararam que são necessárias as precauções de contato frente aos pacientes portadores dessa patologia. Esse resultado foi maior quando comparado aos estudos de Garcia [3], cujo índice encontrado foi de 19,4\%, e de Moreira [35], que apresentou porcentagem de apenas 9\% para essa doença. Vários estudos apontam para a necessidade crescente das práticas adequadas de biossegurança no tratamento desses pacientes, pois o potencial infectante dos vírus que causam essa patologia é grande, sendo o da hepatite B 100 vezes maior quando comparado ao HIV, e o da hepatite C, 10 vezes maior [41]. Dessa forma, a lavagem das mãos, antes e após qualquer procedimento, além do uso de aventais, luvas e máscaras em situações em que o profissional está em contato com sangue, secreções, líquidos corpóreos e excretas, são medidas preconizadas no cuidado aos pacientes portadores da hepatite $[42,43]$. Na prática fisioterapêutica, recomenda-se também o uso dos EPIs e dos EPCs, além de um programa de imunização através da vacinação e a desinfecção e esterilização dos equipamentos utilizados [44].

Quando questionados acerca das precauções de contato para pacientes com meningite, $25,7 \%$ dos estudantes afirmaram que essas práticas são necessárias. Esse resultado foi maior que o encontrado no estudo de Moreira [3], no qual apenas $12 \%$ dos discentes fez tal afirmação; por outro lado, na pesquisa de Garcia [35], 61,3\% dos participantes afirmaram que tais medidas são importantes. Essa discordância entre os resultados demonstram a falta de conhecimento dos diferentes tipos de precaução, visto que diversos estudos apontam que as medidas recomendadas no atendimento a pacientes portadores de meningite seriam a precaução padrão e a precaução para gotículas [35,45].

Por fim, a dengue foi a patologia menos apontada pelos discentes, com apenas $7,6 \%$ de respostas. Pode-se atribuir isso ao fato de ser uma patologia com meio de transmissão bem difundido, principalmente em campanhas de prevenção promovidas pela mídia. Sabe-se, portanto, que as medidas elencadas no cuidado a esses pacientes dizem respeito às precauções padrão $[35,45]$. Dessa forma, é imprescindível que os trabalhadores da saúde conheçam os meios de propagação dos patógenos, pois a falta de conhecimento dessa temática pode resultar no aumento da incidência de infecções entre pacientes e profissionais da área [46-48].

Em relação aos equipamentos de proteção individual (máscara, óculos, jaleco, touca e luvas), devem ser utilizados pelos fisioterapeutas dentro do ambiente hospitalar, independente do risco de exposição a aerossóis [49]. No estudo realizado por Lima, 
todos os participantes informaram que utilizam EPI independente do diagnóstico do paciente, além de possuírem hábito de higienizar as mãos antes e após os atendimentos [50]. Castro [27] constatou que o percentual da adesão à utilização de luvas e aventais foi elevado, com cerca de $91 \%$ de respostas; no presente estudo, o uso de luvas também apresentou índice elevado na identificação como EPI, com 99\% de afirmações. Entretanto, os trabalhos se diferenciam em comparação ao uso dos óculos e aventais, pois no presente estudo, a porcentagem de adesão foi elevada, ao contrário do encontrado por Castro [27] e por Lima [50], de acordo com os quais tais itens foram considerados os itens mais esquecidos. Segundo o estudo de Passos [51], os fisioterapeutas são uma das classes avaliadas mais sujeitas aos riscos ocupacionais, pois o uso de óculos de proteção é um dos mais negligenciados, além do uso de toucas, que foram os únicos EPIs visualizados com baixa adesão. Quanto ao uso de touca, a classe de fisioterapeutas foi a única que descumpriu a medida [50,51].

No que se refere às limitações desta pesquisa, pode-se citar a execução da coleta, a qual foi realizada em uma única instituição de ensino. Todavia, isso não comprometeu o desenvolvimento do estudo, pois houve representatividade entre os discentes de Fisioterapia.

\section{Conclusão}

O elevado percentual de respostas assertivas relacionadas aos conhecimentos sobre biossegurança, no presente estudo, demonstrou que durante a formação acadêmica há o preparo dos estudantes de Fisioterapia sobre segurança ocupacional para a prevenção de contágio por patologias infecciosas. Porém, na comparação com outros trabalhos que avaliaram a adesão de profissionais de saúde a essas medidas, é notório que o aprendizado adquirido durante a formação acadêmica é perdido no decorrer das práticas ocupacionais, o que sinaliza a importância das práticas de educação continuada na prática clínica do profissional fisioterapeuta.

Diante disso, são necessários programas de intervenção com capacitação com maior aproximação da realidade epidemiológica e social e treinamentos específicos sobre a biossegurança durante a graduação e no decorrer da prática clínica. Recomenda-se também que novos estudos relacionados à percepção dessas doenças sejam realizados, o que irá ampliar os conhecimentos dessa temática e embasar futuras medidas referentes à formação desses profissionais.

\section{Conflito de interesses}

As pesquisadoras declaram que não possuem conflitos de interesse.

\section{Financiamento}


O custo da pesquisa foi financiado com recursos próprios das pesquisadoras.

Contribuição dos autores

Planejamento da pesquisa, criação do projeto, coleta de dados, análises estatísticas, construção de gráficos e tabelas, redação do artigo científico: Santos JS, Pereira OC, Santos MM; Análises estatísticas e na revisão do artigo final: Ribeiro LBQ; Orientação na elaboração do projeto, desenvolvimento da coleta, análise dos dados e redação do artigo científico: Medrado ARA

\section{Referências}

1. Ribeiro LCM, Souza ACS, Tipple AFV, Melo DS, Peixoto MKAV, Munari DB. Fatores intervenientes no fluxo de atendimento ao profissional acidentado com material biológico. Rev Esc Enferm USP 2014;48(3):507-13. doi: 10.1590/S0080623420140000300017

2. Oliveira ACD, Paiva MHRS. Análise dos acidentes ocupacionais com material biológico entre profissionais em serviços de atendimento pré-hospitalar. Rev Latinoam Enferm 2013;21(1):309-15. doi: 10.1590/S0104-11692013000100004

3. Moreira MO. Medidas de precaução padrão no ambiente hospitalar adotadas por alunos do curso de fisioterapia [TCC] [Internet]. Campina Grande: Universidade Estadual da Paraíba; 2010. [cited 2022 Jan 19]. Available from: https://shortest.link/Gxz

4. Brasil. Ministério da Saúde. Biossegurança em Saúde: prioridades e estratégias de ação / Ministério da Saúde, Organização Pan-Americana da Saúde. Brasília: Ministério da Saúde; 2010. 242 p. Série B. Textos Básicos de Saúde

5. Oliveira CLBS, Souza FGC, Dias IKR, et al. Manual de biossegurança: serviço de enfermagem. Rev Enferm UFPE on line [Internet]. [cited 2022 Jan 19]. Available from: https://ifce.edu.br/noticias/enfermagem-do-ifce-elabora-manual-debiosseguranca/manual-de-biosseguranca-do-servico-de-enfermagem.pdf

6. Ferreira LL. Estrutura clonal e multirresitência em Pseudomonas aeruginosa [Dissertação] [Internet]. Rio de Janeiro: Instituto Nacional de Controle de Qualidade em Saúde, Fundação Oswaldo Cruz; 2005;113 p. [cited 2022 Jan 19]. Available from: https://www.arca.fiocruz.br/handle/icict/8542

7. Santos FSVD. Biossegurança em hospitais, prevenção e controle de infecções: uma revisão [TCC]. Governador Mangabeira: Faculdade Maria Milza; 2016. http://131.0.244.66:8082/jspui/123456789/240

8. Scheidt KLS, Rosa LRS, Lima EFA. As ações de biossegurança Implementadas pelas comissões de controle de infecções hospitalares. Revista Enferm UERJ [Internet] 2006 [cited 2022 Jan 19];14(3):372-7. Available from: https://www.arca.fiocruz.br/handle/icict/11363

9. Guimarães VC, Estrela CRA, Costa DLS, Mata MC, Silva ET. Comissão de Controle de Infecção Hospitalar: Avaliação da Entrega da Documentação à Vigilância Sanitária. Brazilian Journal of Development 2021;7(7):71105-18. doi: 10.34117/bjdv7n7-336

10. Gomes CAC, Costa JL, Souza Júnior G. O uso de medidas de biossegurança pelos fisioterapeutas. Anais do VII Seminário de Integração Científica da Universidade do 
Estado do Pará. Universidade do Estado do Pará; 2018 Nov 21. doi: 10.31792/21759766.viisic.2018.164

11. Bolick D, Brady C, Bruner DW, Edelstein S, Lane K, McLaughlin MB, et al. Segurança e controle de infecção. Rio de Janeiro: Reichmann \& Affonso; 2000.

12. Campos SF, Vilar MSA, Vilar DA. Biossegurança: conhecimento e adesão às medidas de precauções padrão num hospital. Rev Bras Ciênc Saúde 2011;15(4):415-20. doi: 10.4034/RBCS.2011.15.04.06

13. Fiesp/Ciesp - Federação das Indústrias do Estado de São Paulo; Centro das Indústrias do Estado de São Paulo. Legislação de Segurança e Medicina do Trabalho. Manual Prático. São Paulo; 2003.

14. Gardezi S, Ikram A. Application of biosafety principles in laboratory analysis of clinical samples from patients with COVID-19. J Pak Med Assoc 2020;(0):1. doi: 10.5455/jpma. 10

15. Schroeder MDS, Marin C, Miri F. Biossegurança: grau de importância na visão dos alunos do curso de graduação de Odontologia da Univille. Rev Sul-Bras Odontol [Internet] 2010 [cited 2022 Jan 19];7(1):206.

https://www.redalyc.org/pdf/1530/153012949003.pdf

16. Melo TRNB, Costa PS, Oliveira VS, Diniz MAG, Oliveira Júnior AG. Avaliação do controle das medidas de biossegurança adotadas por acadêmicos de Odontologia. Revista Eletrônica Acervo Científico [Internet]. Revista Eletrônica Acervo Saude; 2020;8:e2112. doi: 10.25248/reac.e2112.2020

17. Xerez JE, Neto HC, Júnior FL, Maia CA, Galvão HC, Gordón-Núñez MA. Perfil de acadêmicos de Odontologia sobre biossegurança. Revista da Faculdade de Odontologia de Porto Alegre 2012;53(1):11-5. doi: 10.22456/2177-0018.31231

18. Cararro TE, Gelbcke FL, Sebold LF, Kempfer SS, Zapelini MC, Waterkemper R. A biossegurança e segurança do paciente na visão de acadêmicos de enfermagem. Rev Gaúcha Enferm 2012;33(3):14-9. doi: 10.1590/s1983-14472012000300002

19. Gasperini L, Stédile MO, Santos MN, Mello MCR, Moraes VM, Margoti FH, et al. Recomendações para o atendimento de pacientes suspeitos ou confirmados de COVID-19 pelos fisioterapeutas no departamento de emergência. Abramed 2020. [cited 2020 Jun 14]. Available from: https://abramede.com.br/recomendacoes-para-oatendimento-de-pacientes-suspeitos-ou-confirmados-de-covid-19-pelos-fisioterapeutasno-departamento-de-emergencia/

20. Tomo S, Boer NP, Correia TM, Silva WR, Lima DP, Cunha-Correia AS. Conhecimento de graduandos em Odontologia a respeito das normas de biossegurança. Arch Health Invest 2014;3(4):9-17. https://archhealthinvestigation.com.br/ArcHI/article/view/789

21. Nobre ARM, Nobre KM, Nobre CVF. Norma Regulamentadora 32 e sua importância para a saúde no ambiente de trabalho. Revista Interdisciplinar de Ciências Médicas Anais [Internet] 2018 [cited 2022 Jan 19];1-7. 
http://www.gpicursos.com/interagin/gestor/uploads/trabalhosfeirahospitalarpiaui/9322eb63f53b07d7974d725225057d74.pdf

22. Silva GS, Almeida AJ, Paula VS, Villar LM. Conhecimento e utilização de medidas de precaução-padrão por profissionais de saúde. Escola Anna Nery [Internet] 2012;16(1):103-10. doi: 10.1590/s1414-81452012000100014

23. Ferreira LA, Peixoto CA, Paiva L, Silva QCG, Rezende MP, Barbosa MH. Adesão às precauções padrão em um hospital de ensino. Rev Bras Enferm 2017;70(1):96-103. doi: 10.1590/0034-7167-2016-0138

24. Hessels AJ, Larson EL. Relationship between patient safety climate and standard precaution adherence: a systematic review of the literature. Journal of Hospital Infection 2016;92(4):349-62. doi: 10.1016/j.jhin.2015.08.023

25. Porto JS, Marziale MHP. Motivos e consequências da baixa adesão às precauções padrão pela equipe de enfermagem. Rev Gaúch Enferm 2016;37(2). doi: 10.1590/19831447.2016.02.57395

26. Oliveira AC, Cardoso CS, Mascarenhas D. Intensive care unit professionals' knowledge and behavior related to the adoption of contact precautions. Rev Latinoam Enferm 2009;17(5):625-31. doi: 10.1590/S0104-11692009000500005

27. Castro AF, Rodrigues MCS. Auditoria de práticas de precauções-padrão e contato em Unidade de Terapia Intensiva. Rev Esc Enferm USP 2019;53. doi: 10.1590/s1980220x2018018603508

28. Oliveira AC, Gonzaga C, Costa R, Damaceno QS, Garbaccio JL. Health care professionals' view of the challenges and perspectives for containing bacterial resistance. Rev Eletrônica Enferm 2013;15(3):747-54. doi: 10.5216/ree.v15i3.19821

29. Alvim ALS, Santos FCR. Medidas de precaução de contato para prevenção e controle de infecções: relato de experiência. Rev Enferm Cent O Min 2017;7:e1333. doi: 10.19175/recom.v7i0.1333

30. Moraes GM, Cohrs FM, Batista REA, Grinbaum RS. Infection or colonization with resistant microorganisms: identification of predictors. Acta Paul Enferm 2013;26(2):18591. doi: 10.1590/S0103-21002013000200013

31. Grota PG, Grant PS. Environmental Infection Prevention. Critical Care Nursing Quarterly [Internet]. Ovid Technologies (Wolters Kluwer Health) 2018;41(1):38-46. doi: 10.1097/cnq.0000000000000184

32. Uneke CJ, Ndukwe CD, Oyibo PG, Nwakpu KO, Nnabu RC, Prasopa-Plaizier N. Promotion of hand hygiene strengthening initiative in a Nigerian teaching hospital: implication for improved patient safety in low-income health facilities. Braz J Infect Dis 2013;18(1):21-7. doi: 10.1016/j.bjid.2013.04.006

33. Portela DA, Mouta AAN, Alves ARR, Almeida FCR, Silva ACB, Lopes PF, et al. A importância da higienização das mãos nas unidades de terapia intensiva: os perigos das infecções relacionadas à assistência à saúde. REAS [Internet]. 2020 [cited 2022 Jan 19]. Available from: https://acervomais.com.br/index.php/saude/article/view/3854 
34. Ministério da Saúde (BR). Agência Nacional de Vigilância Sanitária. Investigação e controle de bactérias multirresistentes [Internet]. Brasília: Ministério da Saúde; 2007. [cited 2018 June 15]. http://anvisa.gov.br/servicosaude/controle/reniss/manual\%20_controle_bacterias.pdf

35. Garcia PN. Adesão dos profissionais de saúde às precauções de contato em unidade de terapia intensiva. [Dissertação] [Internet]. Botucatu: UNESP; 2011. Available from: http://hdl.handle.net/11449/96447

36. Campos SF, Vilar MSA, Vilar DA. Biossegurança: conhecimento e adesão às medidas de precauções padrão num hospital. Rev Bras Ciên Saúde 2011;15(4):415-20. doi: 10.4034/rbcs.2011.15.04.06

37. Brasil. Ministério da Saúde. Doenças relacionadas ao trabalho - Manual de Procedimentos para os Serviços de Saúde: Série A. Normas e Manuais Técnicos; Brasília/DF: n114; 2001.

38. Carmagnani MIS. Segurança e controle de infecção hospitalar. São Paulo: Reichmann e Affonso; 2000.

39. Duarte NS, Mauro MYC. Análise dos fatores de riscos ocupacionais do trabalho de enfermagem sob a ótica dos enfermeiros. Rev Bras Saúde Ocup 2010;35(121):157-67. doi: $10.1590 / S 0303-76572010000100017$

40. Cunha ACD, Valente GSC. Desvelando o Conhecimento dos Trabalhadores de Enfermagem acerca dos Riscos Biológicos na Emergência. Ensino, Saúde e Ambiente [Internet]. Pro Reitoria de Pesquisa, Pós-Graduação e Inovação - UFF 2009;30;2(2). doi: 10.22409/resa2009.v2i2.a21045

41. Ferreira CT, Silveira TR da. Hepatites virais: aspectos da epidemiologia e da prevenção. Rev Bras Epidemiol 2004;7(4):473-87. doi: 10.1590/s1415$790 \times 2004000400010$

42. Gershon RRM, Vlahov D, Felknor SA, Vesley D, Johnson PC, Delcios GL, et al. Compliance with universal precautions among health care workers at three regional hospitals. American Journal of Infection Control 1995;23(4):225-36. doi: 10.1016/01966553(95) $90067-5$

43. Tresoldi AT, Chagas L, Padoveze $C$. Princípios gerais para normatização de isolamentos. In: Colombrine, Mucke AG, Figueiredo RM. Enfermagem em infectologia. São Paulo: Atheneu; 2001.

44. Cantanhêde IGLT. Hepatite B: conhecimento, cobertura vacinal e teste de imunização dos estudantes [TCC] [Internet]. Porto Velho: Centro Universitário São Lucas; 2017. [cited 2022 Jan 19]. Available from: https://shortest.link/Gp9

45. Lacerda MKS, Souza SCO de, Soares DM, Silveira BRM da, Lopes JR. Precauções padrão e precauções baseadas na transmissão de doenças: revisão de literatura. Revista de Epidemiologia e Controle de Infecção 2014;4(4). doi: 10.17058/reci.v4i4.4952 
46. Barros FE, Soares E, Teixeira MLO, Castelo Branco EMS. Controle de infecções a pacientes em precaução de contato. Rev Enferm UFPE on line 2019;19;13(4):1081. doi: 10.5205/1981-8963-v13i4a238991p1081-1089-2019

47. Souza ES, Belei RA, Carrilho CMDM, Matsuo T, Yamada- Ogatta SF, Andrade G, et al. Mortality and risks related to healthcareassociated infection. Texto Contexto-Enferm 2015;24(1):220-8.doi: 10.1590/0104-07072015002940013

48. Siegel JD, Rhinehart E, Jackson M, Chiarello L. 2007 Guideline for isolation precautions: preventing transmission of infectious agents in healthcare settings [Internet]. June 2007 [cited 2022 Jan 19]. Available from: http://www.cdc.gov/ncidod/dhqp/pdf/isolation2007.pdf

49. Matte DL, Cacau L, Reis LFDF, Assis MC. Recomendações sobre o uso de equipamentos de proteção individual (EPIs) no ambiente hospitalar e prevenção de transmissão cruzada na COVID-19. Associação Brasileira de Fisioterapia Cardiorrespiratória e Fisioterapia em Terapia Intensiva 2020;11(Supl1):47. doi: 10.47066/2177-9333.ac20.covid19.005

50. Lima NLC, Silva NA, Oliveira AS. Fatores de risco associados as afecções respiratórias em fisioterapeutas das unidades de terapia intensiva de Caruaru-PE. [TCC] [Internet]. Caruaru: Centro Universitário Tabosa de Almeida; 2019. Available from: http://repositorio.asces.edu.br/handle/123456789/2505

51. Passos BBC, Vasconcelos TB, Bastos VPD, Sousa CT. Desatenção às normas de biossegurança por profissionais de saúde em unidade de terapia intensiva de hospital na cidade de Fortaleza/CE. Rev Saúde Pública [Internet]. 2013 [cited 2022 Jan 19];6(1):35-49. http://www.repositorio.ufc.br/handle/riufc/7927 